\title{
Ethanol and Isopropanol detoxification associated with the Adh locus of Drosophila melanogaster
}

\author{
F. J. S. Sánchez-Cañete, G. Dorado \\ and M. Barbancho*
}

Departamento de Genética, Facultad de Ciencias, Universidad de Córdoba, 14071 Córdoba, Spain.

The detoxification of both ethanol and isopropanol in relation to the Adh locus was studied in the "Lagar de los Reyes" (LR) lines of Drosophila melanogaster. Homozygous lines for the $A d h^{\mathrm{F}}$ and the $A d h^{\mathrm{S}}$ alleles were kept on an ethanol-supplemented medium for about 60 generations. After the selection, adult fiies from these selected lines (LRSeF and LRSeS) were tested for utilisation of, and tolerance to, ethanol and isopropanol, and compared with appropriate controls (LRCF and LRCS) kept on regular medium. Both LRC and LRSe were able to use ethanol (but not isopropanol) as food, the selected flies showing a larger utilisation range than controls. Independently of the lines, FF fies showed a higher or equal ethanol and isopropanol tolerance than SS animals, the females being more or equally tolerant compared with the males. Increased tolerance to ethanol (but not to isopropanol detoxification is equally tolerant compared with the males. Increased tolerance to ethanol (but not to isopropanol) was found in the selected lines. Because of this, a partial independence of ethanol and isoproponal detoxification is suggested.

\section{INTRODUCTION}

The alcohol dehydrogenase (Adh) polymorphism in Drosophila melanogaster was first described by Johnson and Denniston (1964). Since then, a considerable amount of information about the $A d h$ locus has been obtained (see van Delden, 1982, for a review).

The main function of the $\mathrm{ADH}$ isozymes in insect metabolism seems to be the detoxification of environmental alcohols. The $\mathrm{ADH}$ catalyses the oxidation of primary and secondary alcohols to their corresponding aldehydes or ketones respectively, which is associated with a concurrent reduction of $\mathrm{NAD}^{+}$to $\mathrm{NADH}^{+}$(Grell et al., 1968; Dickinson and Sullivan, 1975). In vitro, homozygous FF individuals show an ADH activity at least twice that of SS homozygotes, with heterozygotes (FS) showing intermediate activity values (Gibson, 1970; Ward and Hebert, 1972; Vigue and Johnson, 1973; Day et al., 1974; Ward, 1974, 1975; Oakeshott, 1976 a, b; McDonald and Ayala, 1978; Birley et al., 1981).

Both ethanol tolerance and ethanol utilisation seem to depend on an active ADH (David et al., 1976; David, 1977). However, both of these traits may, at least partially, be controlled by different

\footnotetext{
* To whome correspondence and reprint requests should be
} sent. genetic mechanisms (van Herrewege and David, 1980; Dorado and Barbancho, 1984). In any case, ethanol is transformed into acetaldehyde which is further converted into acetate and thus used as metabolic energy through the Krebs cycle (Clarke, 1975; David et al., 1976; David, 1977; DeltombeLietaert et al., 1979). ADH isozymes seem to have a dual function catalysing both ethanol and acetaldehyde oxidations in D. melanogaster (Heinstra et al., 1983). Ethanol and other primary alcohols can be clearly used as food by $D$. melanogaster, increasing the life span of flies deprived of other food resources (van Herrewege and David, 1974; Deltombe-Lietaert et al., 1979; van Herrewege et al., 1980; Anderson et al., 1981; Dorado and Barbancho, 1984), and even when the flies are exposed only to ethanol vapour (van Herrewege and David, 1978). The survival time of adult $D$. melanogaster flies with ethanol as the only food resource seems to be correlated with the ADH activity (LibionMannaert et al., 1976), FF flies better using the ethanol as metabolic energy than SS flies (Daly and Clarke, 1981; Dorado and Barbancho, 1984).

On the other hand, it is well known that secondary alcohols, like isopropanol, are more toxic than their corresponding primary alcohols (David et al., 1976, 1981). Nevertheless, in vitro, the ADH is much more active on secondary than on primary alcohols as substrates (Vigue and Johnson, 1973; 
Day et al., 1974; Morgan, 1975; Oakeshott, 1976b; Chambers et al., 1978). This higher toxicity (a secondary toxicity) seems to be due to the ketone which is produced from isopropanol (David et al., 1976). Isopropanol and other secondary alcohols are not used as energy sources and they do not extend longevity (van Herrewege et al., 1980). However, the $\mathrm{ADH}$ isozymes must also play a significant role in the detoxification of isopropanol since there has been reported (1) a positive correlation between tolerance to isopropanol and $\mathrm{ADH}$ activity (McDonald and Avise, 1976), (2) an increase of $A d h^{\mathrm{F}}$ frequencies on isopropanol supplemented media (van Delden et al., 1975), and (3) a higher tolerance of $\mathrm{ADH}$-active flies to isopropanol and acetone than $\mathrm{ADH}$-null flies (David et al., 1976, 1981).

In summary, the ADH isozymes seem to play an important role in the detoxification of both primary (ethanol) and secondary (isopropanol) alcohols in D. melanogaster. Hence, it should be interesting to test if an increase in the tolerence to a primary alcohol is associated with a higher tolerance to a secondary one. In the present study a co-selection for increasing both ethanol and isopropanol tolerance, in relation to $A d h$ genotypes and sexes of adult flies, has been tested. For this purpose homozygous lines for the $A d h^{\mathrm{F}}$ and the $A d h^{\mathrm{S}}$ alleles were kept for many generations on both normal and ethanol supplemented media, and the ability to tolerate and/or use both ethanol and isopropanol was tested in both control and selected lines.

\section{MATERIALS AND METHODS}

The flies used came from LR lines, which were founded from a sample collected in the "Lagar de los Reyes" winery of Baena, Córdoba (Spain). Two lines, one homozygous FF and the other homozygous SS, were maintained on normal or regular medium (control lines; LRCF and LRCS), and on ethanol-supplemented medium (selected lines; LRSeF and LRSeS). The number of generations of selection was about 60 . For more details about lines and culture conditions see Dorado and Barbancho (1984).

To test selection efficiency, adult longevity on 10 per cent $\mathrm{v} / \mathrm{v}$ ethanol and 2 per cent $\mathrm{v} / \mathrm{v}$ isopropanol-supplemented media was determined for control and selected lines. For this purpose, samples from LRC and LRSe lines were both grown for one generation on regular food and twoto four-day-old flies from these cultures fed one day more on fresh regular medium. Male and female longevity of three- to five-day-old flies was determined daily in hermetically closed vials (100 mm high $\times 30 \mathrm{~mm}$ diameter), containing $3 \mathrm{ml}$ of the appropriate food. The flies were not etherised and 5 replicates, each vial containing 10 males and 10 females, were made.

To test adult tolerance to both ethanol and isopropanol, alcohol solutions of either ethanol $(0,1 \cdot 25,2 \cdot 5,5,7 \cdot 5,10,12 \cdot 5$ and 15 per cent $\mathrm{v} / \mathrm{v})$ or isopropanol $(0,1,2,3,4$ and 5 per cent $\mathrm{v} / \mathrm{v})$ were used. Mortality measurements were determined in the same way as indicated above, but $3 \mathrm{ml}$ of the alcohol solutions were dropped on to $0.4 \mathrm{~g}$ of absorbent cotton, standing at the bottom of the vials, on the top of which a circle of Whatman no. 3 filter paper with the same diameter of the vial was placed. Each vial contained 10 males and 10 females and 3 replicates for each alcohol concentration were made.

All the experiments were carried out at approximately $25^{\circ} \mathrm{C}$.

\section{RESULTS}

\section{(i) Adult longevity on ethanol-supplemented medium}

As a selection control, adult longevity in 10 per cent ethanol-supplemented medium was determined. Fig. 1 shows the longevities of control and selected lines of the two Adh genotypes on normal and ethanol-supplemented medium as the relationship between adult mortalities and days of exposure.

Comparing the longevity on 10 per cent ethanol medium with that on the normal one a clear toxic effect is apparent, though such a toxic effect is higher for the SS flies than the FF ones. On the other hand, it has been an efficient ethanol selection since the ethanol tolerance of both selected lines (LRSeF and SRSeS) has improved. After selection the time taken for 50 per cent of FF and SS flies to die was $7 \cdot 0$ and $6 \cdot 0$ days respectively. Comparatively, flies of the same genotype from the control lines took $2 \cdot 7$ and 0.8 days.

A factorial ANOVA (summarised in table 1) was used to quantify the extent of the ethanol tolerance on lines, genotypes and sexes. In this analysis, angular transformations $(\arcsin \sqrt{p})$ of mortality at the third day (day with a high mortality at control test) were used. As expected, significant differences between lines were observed, which indicate that the LRSe lines have a higher ethanol 


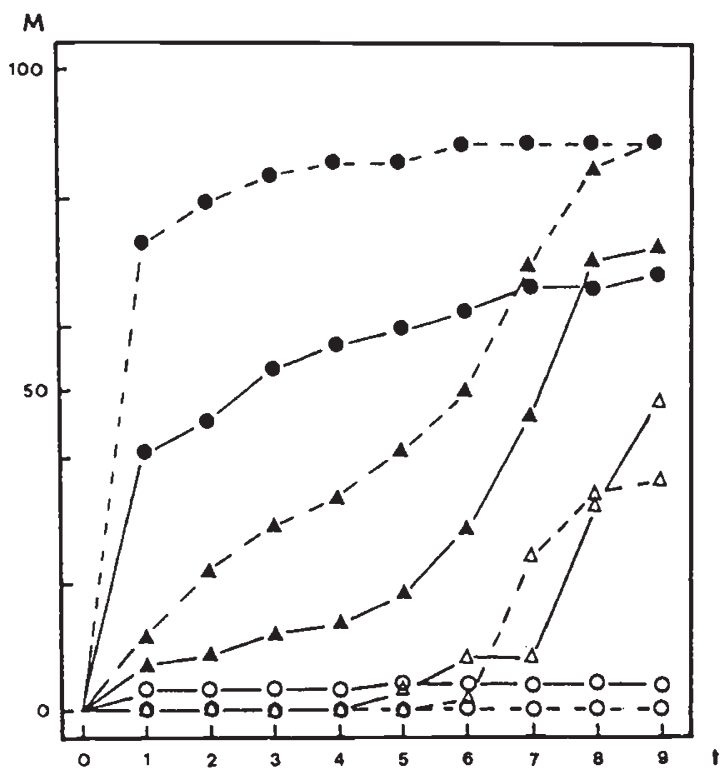

Figure 1 Adult longevity as a relationship between adult mortality (M) and the number of days' exposure (t), for LRC $(O, \mathcal{O})$ and $\operatorname{LRSe}(\triangle, \Delta)$ lines on non-supplemented $(O, \triangle)$ and a 10 per cent ethanol supplemented medium $(\mathbf{O}, \mathbf{\Delta})$. FF: $-;$ SS: - - -

tolerance than the LRC ones. Significant intergenotypic differences were also detected, presumably resulting from the higher tolerance of the FF flies.

\section{(ii) Adult longevity on ethanol solutions}

Once the effect of the selection on improving the ethanol tolerance was corroborated, adult longevity on ethanol or isopropanol solutions was determined. Fig. 2 shows the mortalities of control and selected lines of both FF and SS genotypes on the different ethanol concentrations as a function of the number of days exposure.

Comparing in fig. 2 adult mortalities on each ethanol concentration to those on distilled water ( 0 per cent), the ability to use ethanol as food can be suggested. For both FF and SS control flies an increase in longevity is observed for smaller concentrations $(1 \cdot 25,2 \cdot 5$ and 5 per cent $)$, this increase being also observed for FF flies at a 7.5 per cent concentration. On the other hand, the ethanol selection increased the adult longevity, since the FF and SS selected flies increased also their longevities at concentrations of 10 per cent and $7 \cdot 5$ per cent respectively. The most likely explanation of these longevity increases seems to be the ability of the flies to use the ethanol as a metabolic energy, this ability having been improved by the ethanol selection.

These observations are statistically supported on the upper part of table 2 , which shows independent mortalities at the third day of treatment, for LRC and LRSe males and females on the different ethanol concentrations. Comparing adult mortalities on each concentration to those on distilled water ( 0 per cent) by means of a Student's test (once angular transformations of mortalities were done), both ethanol utilisation and ethanol toxicity were tested. Some specific differences between males and females can be seen, since the females from LRC lines showed a lower tolerance and lesser utilisation than their respective males. On the other hand, both the ethanol utilisation and the ethanol tolerance have been improved by the selection only in the females.

The lower part of table 2 shows selection, genotypic and sex effects on the different ethanol concentrations used. The selection effect was obtained comparing (by means of a Student's test)

Table 1 Factorial analysis of variance (ANOVA) of the effects of line, genotype and sex on the adult mortality at the third day (transformed to angles) on medium supplemented with 10 per cent ethanol and 2 per cent isopropanol

\begin{tabular}{|c|c|c|c|c|c|}
\hline \multirow{2}{*}{$\begin{array}{l}\text { Source of } \\
\text { variation }\end{array}$} & \multirow[b]{2}{*}{ d.f. } & \multicolumn{2}{|l|}{ Ethanol } & \multicolumn{2}{|c|}{ Isopropanol } \\
\hline & & Deviance & $\mathrm{F}$ & Deviance & $\mathrm{F}$ \\
\hline Lines $(\mathrm{L})$ & 1 & 13596.97 & $35 \cdot 82 \dagger$ & $40 \cdot 55$ & $0.19 \mathrm{~ns}$ \\
\hline $\operatorname{Sex}(S)$ & 1 & 1.70 & $0.00 \mathrm{~ns}$ & $7844 \cdot 80$ & $36.93 \dagger$ \\
\hline Genotypes (G) & 1 & $3108 \cdot 26$ & $8 \cdot 19 \dagger$ & $6677 \cdot 67$ & $31 \cdot 44 \dagger$ \\
\hline $\mathrm{L} \times \mathrm{S}$ & 1 & $472 \cdot 20$ & $1 \cdot 24 \mathrm{~ns}$ & $972 \cdot 22$ & $4 \cdot 58^{*}$ \\
\hline $\mathrm{L} \times \mathrm{G}$ & 1 & $280 \cdot 52$ & $0.74 \mathrm{~ns}$ & $81 \cdot 34$ & $0.38 \mathrm{~ns}$ \\
\hline $\mathrm{S} \times \mathrm{G}$ & 1 & $1965 \cdot 10$ & $5 \cdot 18^{*}$ & $649 \cdot 12$ & $3.06 \mathrm{~ns}$ \\
\hline $\mathrm{L} \times \mathrm{S} \times \mathrm{G}$ & 1 & $1586 \cdot 43$ & $4 \cdot 18^{*}$ & $41 \cdot 58$ & $0.20 \mathrm{~ns}$ \\
\hline Residual & 32 & $379 \cdot 62$ & & $212 \cdot 42$ & \\
\hline Total & 39 & & & & \\
\hline
\end{tabular}

* $P<0.05 ; \dagger P<0.01 ;$ ns $P>0.05$ 

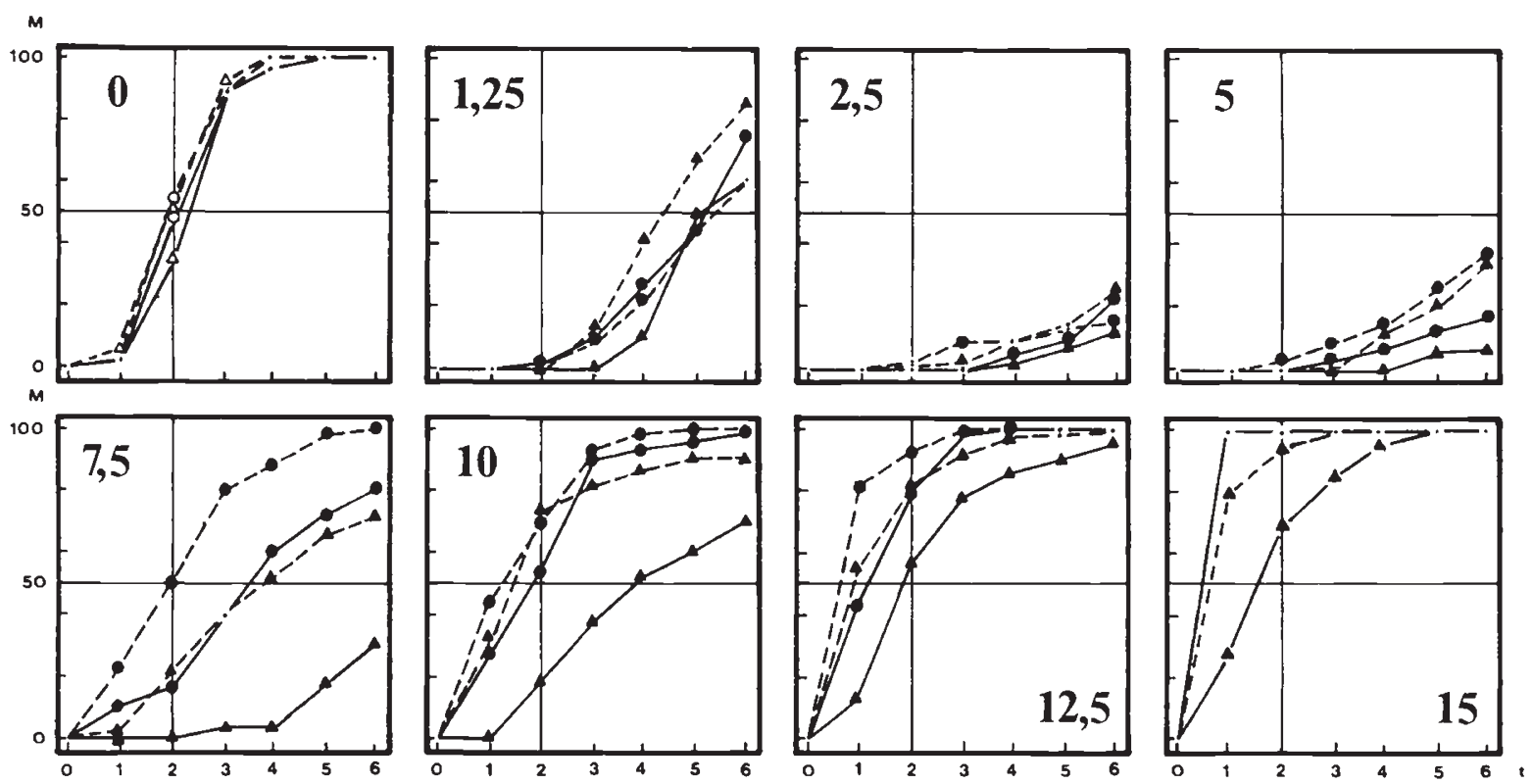

Figure 2 Adult longevity as a relationship between adult mortality and the number of days' exposure for LRC and LRSe lines on different ethanol solutions. Symbols: see fig. 1 .

the mortalities (after angular transformations) between selected and control lines for each sex, genotype and concentration. In general, the selection has only improved the ethanol tolerance of the females at concentrations of 7.5 and above.

The genotypic effects were obtained in the same way as before-by comparing the mortalities of FF vs. SS flies for each sex, line and concentration. Both sexes of FF flies from LRSe lines were more tolerant to ethanol than SS animals at concentrations of at least 7.5 per cent. In contrast, both sexes of FF flies from LRC lines only show a greater ethanol tolerance than the SS ones at concentrations of 7.5 per cent.

The sex effect was obtained comparing females and males of each genotype, line and concentration. Although some differences were seen, in general these were again consistent only for concentrations higher or equal to 7.5 per cent, and only for LRSe lines.

\section{(iii) Adult longevity on isopropanol solutions}

Fig. 3 shows the longevities of the flies from both LRC and LRSe lines, of both FF and SS genotypes, on the different isopropanol concentrations. The ability to use isopropanol as food is also shown in fig. 3 and in the upper part of table 3 . Isopropanol, at concentrations of 1 per cent and above, do not increase the longevity of
D. melanogaster flies from LR lines, independently of the genotype and sex. D. melanogaster is not able to use isopropanol as food. This alcohol is toxic at concentrations of 2 per cent, except for FF females which do tolerate a concentration of 2 per cent. Isopropanol at 1 per cent is well tolerated but does not increase the longevity of the flies. As can be seen in the upper part of table 3, the toxic effect of isopropanol affects both females and males. On the other hand, ethanol selection seems not to have improved the ability to use isopropanol as food.

The interactions of selection, genotype and sex with the different isopropanol concentrations are shown in table 3 (lower part). In general, the selection for improving the ethanol tolerance has not increased isopropanol tolerance of the control lines. However, intergenotypic differences-FF being more tolerant than SS for both control and selected lines-are clearly observed at concentrations of 2 per cent.

Finally, the females showed a greater isopropanol tolerance than the males, particularly at concentrations of 2 per cent, these differences being more apparent in the FF flies.

\section{(iv) Adult longevity on isopropanol- supplemented medium}

To corroborate that the selection to improve the ethanol tolerance had not modified the 
Table 2 Upper: Mortalities at the third day of flies from each line, genotype and sex, and ethanol utilisation/toxicity by comparing the mortalities on each ethanol concentration with that on water $(0$ per cent $)$. ( + : utilised; $-:$ toxic; $=$ : Not utilised and not toxic concentrations).

Lower: Selection, genotypic and sex effects at each ethanol concentration. (Mortalities of LRSe, FF or $\$$ lower $(+)$, higher $(-)$ or similar $(=)$ than that of LRC, SS or ot respectively).

Comparison of mortalities (transformed to angles) using Student's test. Significances: $P<0.05$

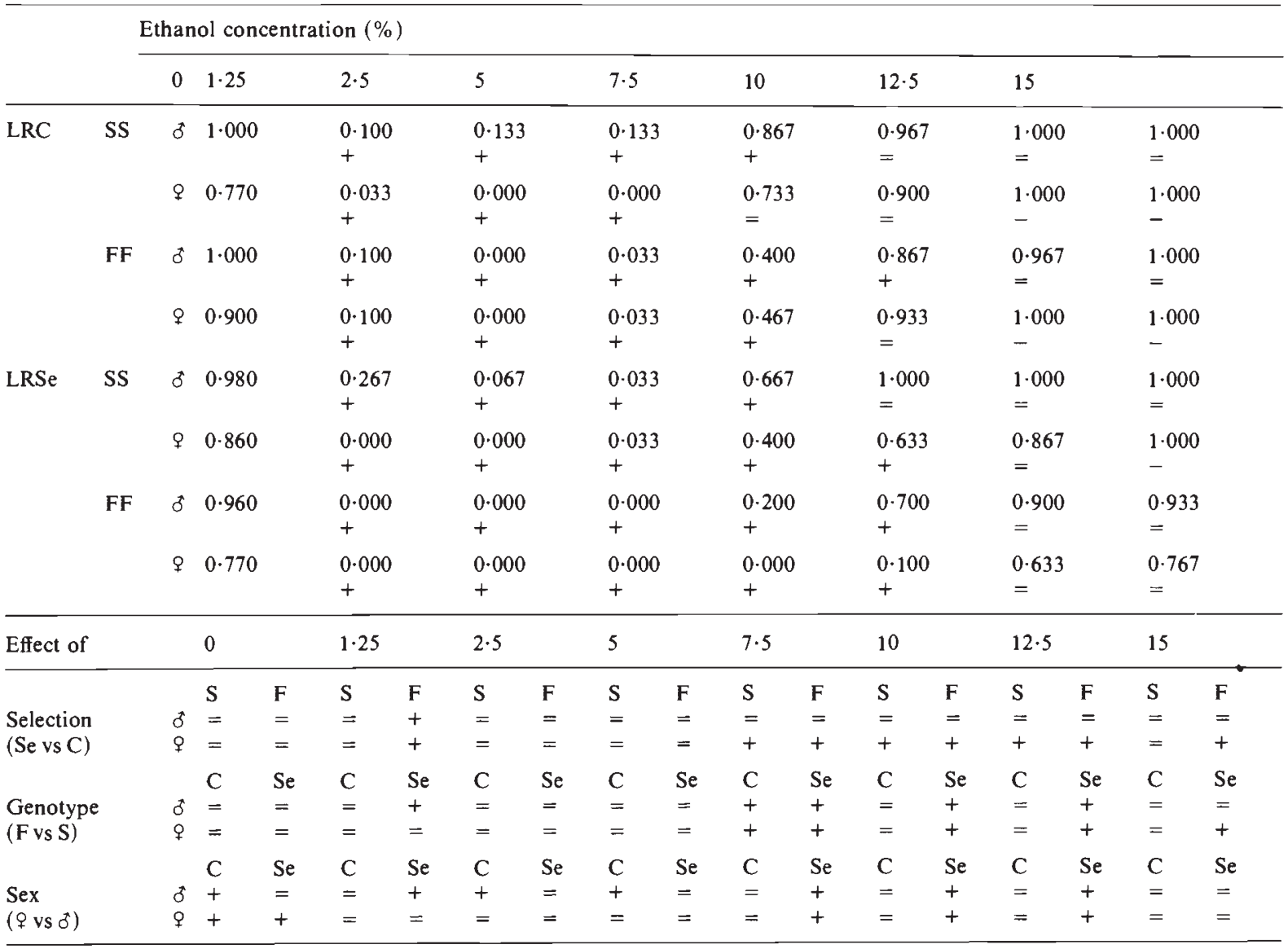

isopropanol tolerance, adult longevities on regular medium supplemented with a 2 per cent isopropanol were determined.

Fig. 4 shows the mean mortalities of males and females on isopropanol-supplemented medium for both LRC and LRSe lines, and for both FF and SS genotypes, as a function of the days of treatment. Although the FF flies are more tolerant to isopropanol than the SS ones, control and selected flies from a same genotype seem to show a similar isopropanol tolerance. This is statistically supported by the factorial ANOVA summarised in table 1 .

\section{DISCUSSION}

Our results are clearly consistent with the well known phenomenon that $D$. melanogaster adults can use primary alcohols as a source of metabolic energy (van Herrewege and David, 1974, 1980; Deltombe-Lietaert et al., 1979; Anderson et al., 1981; Dorado and Barbancho, 1984).

Ethanol, at small and moderate concentrations, extend longevity of $D$. melanogaster adults. In general, our data indicate that non-selected flies are able to use ethanol as food at concentrations up to 7.5 per cent, the FF flies using such 7.5 per cent ethanol concentration more efficiently than the SS flies. Moreover, this ability to use the ethanol is improved, in both FF and SS flies, after a programme of selection for increasing tolerance to ethanol: in general, ethanol-selected flies are able to use concentrations up to 10 per cent ethanol (see fig. 2 and table 2). It is interesting to note that at high concentrations $(7 \cdot 5$ and 10 per cent) only the females, for both Adh genotypes, have improved their ability to use the ethanol as food 

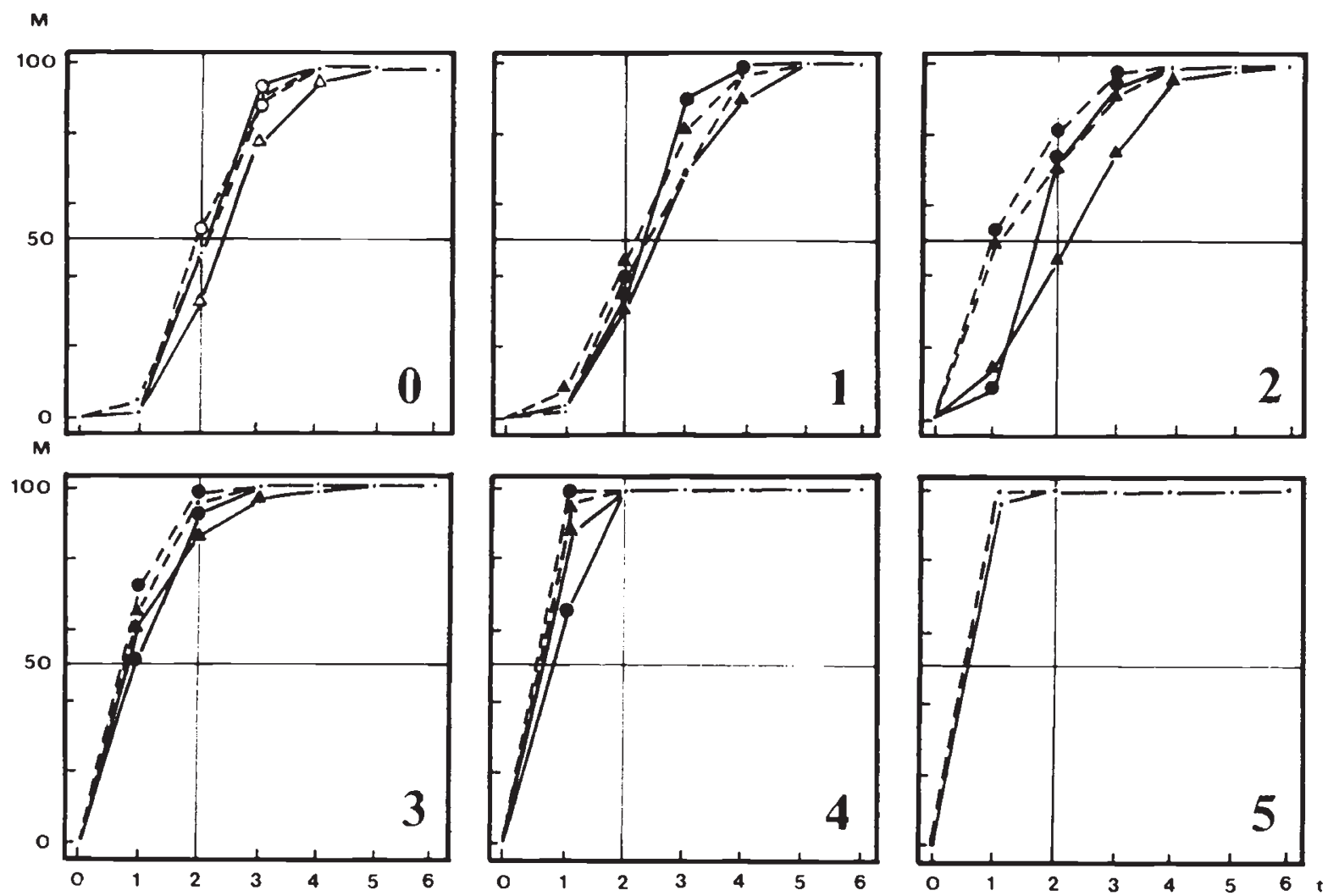

Figure 3 Adult longevity as a relationship between adult mortality and the number of days' exposure for LRC and LRSe lines on different isopropanol solutions. Symbols: see fig. 1.

(table 2).

This ability to use ethanol as food, increasing the survival time of the flies, appears to be positively correlated with the ADH enzymatic activity (Libion-Mannaert et al., 1976). This positive correlation seems to be supported by the facts that 1) the FF flies show a better ability than SS flies to use ethanol as food (Daly and Clarke, 1981; Dorado and Barbancho, 1984; present paper), and 2) ADH-active flies are able to use the ethanol better than ADH-negative flies (van Herrewege and David, 1974, 1978, 1980; David et al., 1981).

Our results also show that in the LR lines the ability to detoxify the ethanol at concentrations not used as food ( $>7.5$ per cent at LRC and $>10$ per cent at LRSe) appears again to be greater in the FF flies than in the SS ones, although only in the selected flies (fig. 2 and table 2). In the nonselected flies this intergenotypic effect only appears to be significant at the concentration of 7.5 per cent (lower part of table 2). Ethanol at $7 \cdot 5$ per cent seems to be a threshold concentration below which the FF and SS flies show a similar ability to use ethanol as food, and above which both exhibit a similar ability to detoxify it. Several studies indicate that FF flies show greater survival than SS animals at high concentrations of ethanol; i.e., more than 10 per cent (Briscoe et al., 1975; Morgan, 1975; van Delden et al., 1978; Dorado and Barbancho, 1984). Such superiority has been associated by some authors with a more active ADH-F isozyme (Gibson and Miklovich, 1971; LibionMannaert et al., 1976; Kamping and van Delden, 1978; van Delden and Kamping, 1983). However, at such high concentrations we have only observed this intergenotypic difference in the selected lines, since FF and SS flies from non-selected lines show a similar ethanol tolerance (lower part of table 2). These non-intergenotypic differences in nonselected flies contrast with the significnt differences observed on the 10 per cent ethanol supplemented medium (fig. 1). This is in agreement with previous observations (Dorado and Barbancho, 1984) in which intergenotypic differences in both LRC and LRSe were seen, using an 11 per cent ethanolsupplemented agar. A different ethanol vapour pressure, higher at ethanol solutions, could be the explanation since Drosophila can utilise ethanol taken in during respiration (Starmer et al., 1977; 
Table 3 Legend similar to that of table 2, but using isopropanol as the testing alcohol. Mortalities checked at the first day of exposure

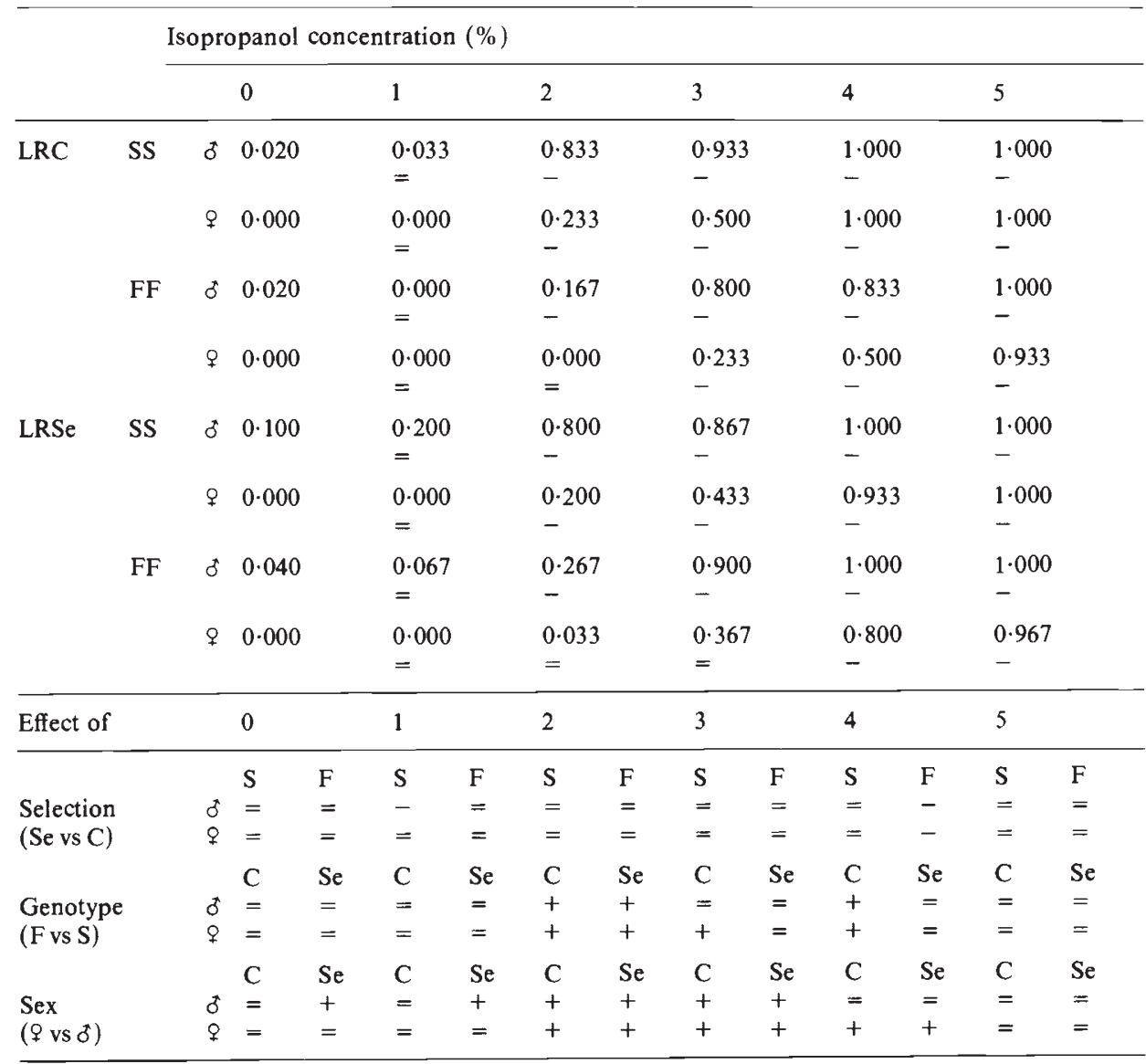

van Herrewege and David, 1978; Ziolo and Parsons, 1982).

On the other hand, at ethanol concentrations higher or equal to $7 \cdot 5$ per cent, the ability of both $\mathrm{FF}$ and SS flies to tolerate the ethanol has only been appreciably improved by the selection in females (lower part of table 2). An increased tolerance to ethancl by selection has been associated by some investigators to a higher $\mathrm{ADH}$ activity of the selected lines (McDonald et al., 1977; Ayala and McDonald, 1980). However, other studies suggest that the ADH per se is not the only factor involved in alcohol tolerance, and that an increased tolerance to ethanol could depend on loci other than Adh (McKenzie and Parsons, 1974; Gibson et al., 1979; Oakeshott, 1979; Ziolo and Parsons, 1982; van Delden and Kamping, 1983).

Our results also demonstrate that at low ethanol concentrations (under 7.5 per cent), neither the genotype nor selection show differential effects on the detoxification of ethanol (lower part of table
2). If the $A d h$ locus is directly involved in ethanol detoxification, the presumed $\mathrm{ADH}$ activity differences between FF and SS flies, and between control and selected lines, seem not to be relevant at concentrations lower than 7.5 per cent. Thus, our findings seem not to agree with those of Daly and Clarke (1981), since they observed differential survival of FF and SS adults maintained on low concentrations of ethanol ( 2 per cent).

Independently of sex, genotype or ethanol selection, D. melanogaster adults are not able to use isopropanol as food, which is toxic at concentrations equal or higher to 2 per cent (fig. 3 and table 3 ). Our results are consistent with the fact that while primary alcohols are used as a source of metabolic energy, secondary alcohols are not (van Herrewege et al., 1980); David et al., 1981). The high toxicity of isopropanol probably results from the acetone derived from it. However, flies with a higher ADH activity are able to tolerate better either isopropanol or acetone than flies with a lower ADH activity (David et al., 1976, 1981; 


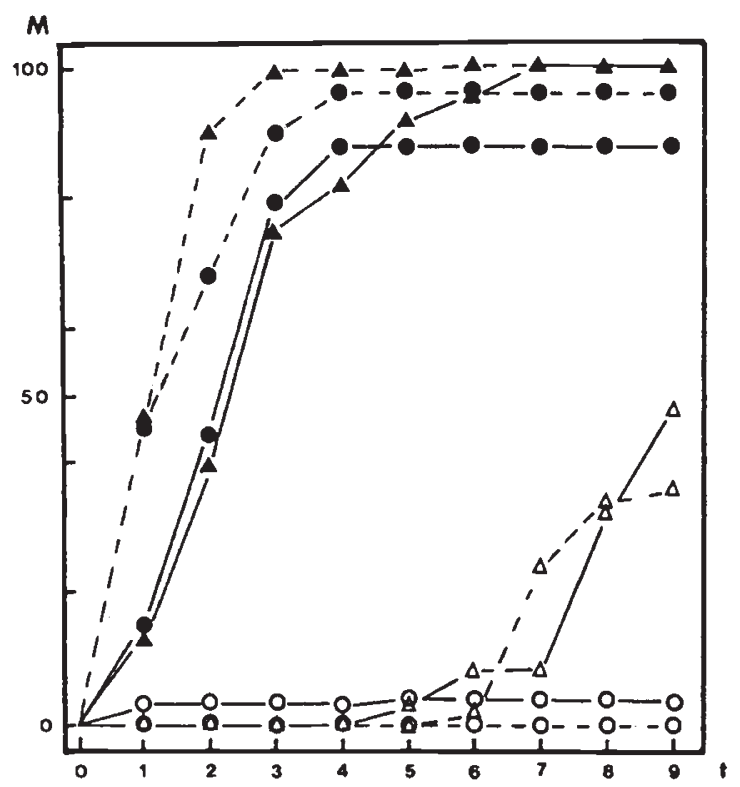

Figure 4 Adult longevity as a relationship between adult mortality and the number of days' exposure for LRC and LRSe lines on non-supplemented and a 2 per cent isopropanol supplemented medium. Symbols: see Fig. 1.

McDonald and Avise, 1976). Our results support this observation mainly at concentrations of 2 per cent; the FF flies from both selected and nonselected lines show higher ability than the SS flies to detoxificate the isopropanol.

As pointed out by David et al. (1981), detoxification of isopropanol by the ADH leads to a physiological paradox: $\mathrm{ADH}$ increases the tolerance to isopropanol in spite of the fact that this enzyme transforms such alcohol into acetone, a much more toxic product. Moreover, both acetone (Papel et al., 1979) and isopropanol (Schwartz and Sofer, 1976) rapidly decrease the ADH activity in vivo. Probably, other loci apart from the Adh locus-but perhaps linked to itcould be involved in the detoxification of secondary alcohols.

In summary, $\mathrm{ADH}$ seems to play a role in the detoxification of ethanol and probably also of isopropanol; FF flies show a higher or equal tolerance to both alcohols than SS flies. However, while selection for increasing tolerance to ethanol appears to be successful at concentrations of at least $7 \cdot 5$ per cent, such selection did not suceed in improving isopropanol tolerance. It is interesting to note the specificity of the selection in our lines, since only the ability to detoxify the selected alcohol was improved. This specific selection was not observed by van Delden and Kamping (1983).
They reported that lines successfully selected for increased tolerance to hexanol, also proved to be more tolerant to other alcohols. However, these authors only tested primary alcohols: hexanol, ethanol and propanol, and the factors involved in the increased tolerances to such alcohols seem not to be the same. While ethanol tolerance appears to be associated with a higher ADH activity, this seems not to be the main factor in the tolerance to hexanol and propanol.

In conclusion, an increase in the tolerance and metabolic utilisation of ethanol, produced through a selection for increasing tolerance to a primary alcohol (ethanol), is not associated with an increase in the tolerance and/or utilisation of a secondary alcohol (isopropanol). These results suggest that different methods of detoxification exist for primary and secondary alcohols in $D$. melanogaster, although both could depend on the enzymatic activity of $\mathrm{ADH}$.

\section{REFERENCES}

ANDERSON, S. M., MCDONALD, J. F. AND SANTOS, M. 1981. Selection at the Adh locus in Drosophila melanogaster: Adult survivalship-mortality in response to ethanol Experientia, 37, 463-464.

AYAlA, F. J. AND MCDONALD, J. F. 1980. Continuous variations: Possible role of regulatory genes. Genetica, $52 / 53$, 1-15.

BIRLEY, A. J., COUCH, P. A. AND MARSON, A. 1981. Genetical variation for enzyme activity in a population of Drosophila melanogaster. VI. Molecular variation in the control of alcohol dehydrogenase (ADH) activity. Heredity, 47, 185196.

BRISCOE, D. A., ROBERTSON, A. AND MALPICA, J. M. 1975. Dominance at $A d h$ locus in response of adult Drosophila melanogaster to environmental alcohol. Nature, 255, 148 149.

CHAMBERS, G. K., MCDONALD, J. F., MCELRESH, M. AND AYALA, F. 1978. Alcohol oxidizing enzymes in 13 Drosophila species. Biochem. Genet., 16, 757-767.

CLARKE, B. 1975. The contribution of ecological genetics to evolutionary theory: detecting the direct effects of selection on particular polymorphic loci. Genetics, 79, 101-113.

DALY, K. AND CLARKE, B. 1981. Selection associated with the alcohol dehydrogenase locus in Drosophila melanogaster: differential survival of adults maintained on low concentrations of ethanol. Heredity, 46, 219-226.

DAVID, J. 1977. Signification d'un polymorphisme enzymatique: la déshydrogénase alcoolique chez Drosophila melanogaster. Ann. Biol., 16, 451-472.

DAVID, J. R., BOCQUET, C., ARENS, M. F. AND FOUILLET, P. 1976. Biological role of alcohol dehydrogenase in the tolerance of Drosophila melanogaster to aliphatic alcohols: utilization of an ADH-null mutant. Biochem. Genet., 14, 989-997.

DAVID, J. R., HERREWEGE, J. VAN, SCHEEMAEKER-LOUIS, M. DE AND PLA, E. 1981. Drosophila alcohol dehydrogenase: detoxification of isopropanol and acetone, substances not used in energy metabolism. Heredity, 47, 263-268. 
DAy, T. H., hillier, P. C. AND Clarke, B. C. 1974. Properties of genetically polymorphic isozymes of alcohol dehydrogenase in Drosophila melanogaster. Biochem. Genet., 11, 141-153.

DELDEN, W. VAN 1982. The alcohol dehydrogenase polymorphism in Drosophila melanogaster. Selection at an enzyme locus. Evolutionary Biology, 15, 187-222.

DELDEN, W. VAN, BOEREMA, A. C. AND KAMPING, A. 1978. The alcohol dehydrogenase polymorphism in populations of Drosophila melanogaster. I. Selection in different environments. Genetics, 90, 161-191.

DELDEN, W. VAN AND KAMPING, A. 1983. Adaptation to alcohols in relation to the alcohol dehydrogenase locus in Drosophila melanogaster. Ent. Exp. and Appl., 33, 97-102.

DELDEN, W. VAN, KAMPING, A. AND DIJK, H. VAN 1975. Selection at the alcohol dehydrogenase locus in Drosophila melanogaster. Experientia, 31, 418-420.

DELTOMBE-LIETAERT, M. C., DELCOUR, J., LENELlEMONFORT, N. AND ELENS, A. 1979. Ethanol metabolism in Drosophila melanogaster. Experientia, 35, 579-581.

DICKINSON, W. J. AND SUllivan, D. T. 1975. Gene-enzyme systems in Drosophila. Springer-Verlag, Berlin, West Germany.

DORADO, G. AND BARBANCHO, M. 1984. Differential responses in Drosophila melanogaster to environmental ethanol: modification of fitness components at the Adh locus. Heredity, 53, 309-320.

GIBSON, J. 1970. Enzyme flexibility in Drosophila melanogaster. Nature, 227, 959-960.

GIBSON, J. B., LEWIS, N., ADENA, M. A. AND WILSON, S. R. 1979. Selection for ethanol tolerance in two populations of Drosophila melanogaster segregating alcohol dehydrogenase allozymes. Aust. J. Biol. Sci., 32, 387-398.

GIBSON, J. B. AND MIKLOVICH, R. 1971. Modes of variation in alcohol dehydrogenase in Drosophila melanogaster. Experientia, 27, 99-100.

GRELL, E. H., JACOBSON, B. K. AND MURPHY, J. B. 1968. Alterations of genetic material for analysis of alcohol dehydrogenase isozymes of Drosophila melanogaster. Ann. N. Y. Acad. Sci., 151, 441-455.

HEINSTRA, P. W. H., EISSES, K. TH., SCHOONEN, W. G. E. J., ABEN, W., WINTER, A. J. DE, HORST, D. J. VAN DER, MARREWIJK, W. J. A. VAN, BEENAKKERS, A. M. TH. SCHARLOO, W. AND THÖRIG, G. E. W. 1983. A dual function of alcohol dehydrogenase in Drosophila. Genetica, $60,129-137$.

HERREWEGE, J. VAN AND DAVID, J. 1974. Utilisation de l'alcohol éthylique dans le métabolisme énergétique d'un insecte: influence sur la durée de survie des adultes de Drosophila melanogaster. C. R. Acad. Sci. Paris, 279, 335338.

HERREWEGE, J. VAN AND DAVID, J. R. 1978. Feeding an insect through its respiration: assimilation of alcohol vapors by Drosophila malanogaster adults. Experientia, 34, 163-164.

HERREWEGE, J. VAN AND DAVID, J. R. 1980. Alcohol tolerance and alcohol utilization in Drosophila: partial independence of two adaptative traits. Heredity, 44, 229-235.

HERREWEGE, J. VAN, DAVID, J. R., AND GRANTHAM, R. 1980. Dietary utilization of aliphatic alcohols by Drosophila. Experientia, 36, 846-847.

JOHNSON, F. M. AND DENNISTON, C. 1964. Genetic variation of alcohol dehydrogenase in Drosophila melanogaster. Nature, 204, 906-907.
KAMPING, A. AND DELDEN, W. VAN 1978. Alcohol dehydrogenase polymorphism in populations of Drosophila melanogaster. II. Relation between ADH activity and adult mortality. Biochem. Genet., 16, 541-551.

LIBION-MANNAERT, M., DELCOUR, J., DELTOMBE-LIETAERT, M. C., LENELLE-MONTFORT, N. AND ELENS, A. 1976. Ethanol as a "food" for Drosophila melanogaster: Influence of the ebony gene. Experientia, 32, 22-24.

MCDONALD, J. F. AND AVISE, J. C. 1976. Evidence for the adaptive significance of enzyme activity levels: interspecific variation in $\alpha-\mathrm{GPDH}$ and ADH in Drosophila. Biochem. Genet., 14, 247-355.

MCDONALD, J. F. AND AYALA, F. J. 1978. Genetic and biochemical basis of enzyme activity variation in natural populations. I. Alcohol dehydrogenase in Drosophila melanogaster. Genetics, 89, 371-388.

MCDONALD, J. F., CHAMBERS, G. K., DAVID, J. AND AYALA, F. J. 1977. Adaptative response due to changes in gene regulation: a study with Drosophila. Proc. Natl. Acad. Sci. $U S A, 74,3462-3466$.

MCKENZIE, J. A. AND PARSONS, P. A. 1974. Microdifferentiation in a natural population of Drosophila melanogaster to alcohol in the environment. Genetics, 77, 385-394.

MORGAN, P. 1975. Selection acting directly on an enzyme polymorphism. Heredity, 35, 124-127.

OAKESHOTT, J. G. 1976a. Selection at the alcohol dehydrogenase locus in Drosophila melanogaster imposed by environmental ethanol. Genet. Res., 26, 265-274.

OAKESHOTT, J. G. 1976b. Biochemical differences between alcohol dehydrogenases of Drosophila melanogaster. Aust. J. Biol. Sci., 29, 365-373.

OAKESHOTT, J. G. 1979. Selection affecting enzyme polymorphisms in laboratory populations of Drosophila malanogaster. Oecologia, 143, 341-354.

PAPEL, I., HENDERSON, M., HERREWEGE, J. VAN, DAVID, J. AND SOFER, W. 1979. Drosophila alcohol dehydrogenase activity in vitro and in vivo: effects of acetone feeding. Biochem. Genet., 17, 553-563.

SCHWARTZ, M. AND SOFER, W. 1976. Diet-induced alterations in distribution of muitiple forms of alcohol dehydrogenase in Drosophila. Nature, 263, 129-131.

STARMER, W. T., HEED, W. B. AND ROCKWOOD-SLUSS, E. S. 1977. Extension of longevity in Drosophila mojavensis by environmental ethanol: differences between subraces. Proc. Natl. Acad. Sci. USA, 74, 387-391.

VIGUE, C. L. AND JOHNSON, F. M. 1973. Isozyme variability in species of the genus Drosophila. VI. Frequency-propertyenvironmental relationships of allelic alcohol dehydrogenase in D. melanogaster. Biochem. Genet., 9, 313227.

WARD, R. D. 1974. Alcohol dehydrogenase in Drosophila melanogaster: activity variation in natural population. Biochem. Genet., 12, 449-458.

WARD, R. D. 1975. Alcohol dehydrogenase activity in Drosophila melanogaster: a quantitative character. Genet. Res. Camb., 26, 81-93.

WARD, R. D. AND HEBERT, P. D. N. 1972. Variability of alcohol dehydrogenase activity in a natural population of Drosophila melanogaster. Nat. New Biol., 236, 243-244.

Z1OLO, L. K. AND PARSONS, P. A. 1982. Ethanol tolerance, alcohol-dehydrogenase activity and $A d h$ allozymes in Drosophila melanogaster. Genetica, 57, 231-237. 